\title{
Novel thin film lift-off process for in situ TEM tensile characterization
}

\author{
Krishna Kanth Neelisetty ${ }^{1}$, Shyam Kumar $\mathrm{CN}^{2}$, Ankush Kashiwar ${ }^{3}$, Torsten Scherer ${ }^{4}$, VS Kiran
} Chakravadhanula $^{5}$ and Christian Kuebel ${ }^{6}$

${ }^{1}$ Thermofisher Scientific, Brno, Jihomoravsky kraj, Czech Republic, ${ }^{2}$ Institute of Nanotechnology, Karlsruhe Institute of Technology, United States, ${ }^{3}$ Institute of Nanotechnology, Karlsruhe Institute of Technology, Belgium, ${ }^{4}$ Institute of Nanotechnology, Karlsruhe Institute of Technology, BadenWurttemberg, Germany, ${ }^{5}$ Institute of Nanotechnology, Karlsruhe Institute of Technology, Telangana, India, ${ }^{6}$ Karlsruhe Institute of Technology, Eggenstein-Leopoldshafen, Baden-Wurttemberg, Germany

Recent advances in MEMS based devices for in situ transmission electron microscopy (TEM) are opening exciting new directions in nanoscale research. The possibilities to combine various stimuli like biasing, heating, etc inside the TEM to study various applied materials e.g., solid state batteries, non-volatile memories, sensors, etc. are continuously increasing, but the challenges to prepare suitable specimens are increasing at the same time. The majority of samples used for in situ TEM experiments are prepared using Focused Ion Beam (FIB). Classic lamella preparation has been standardized and almost automated in previous years. However, unconventional methods are being developed to address the research challenges in sample preparation for in situ studies, e.g. to reduce dose and contamination. This study presents a method for successful FIB lift out from a heating membrane of commercially available MEMS based in situ heating chips onto the push-to-pull (PTP) device (Bruker) for in situ mechanical measurements, minimizing ion beam damage and contamination of the area of interest.

Thin free-standing polymer films were deposited by spin-coating on a MEMS-based in situ heating device (Aduro from Protochips) and nanocrystalline graphene (ncg) was formed across the holes of the heating region as described elsewhere [1,2]. The area of interest for the lift-out is the hole across which the ncg is formed. The heating region in the Aduro device is a free-standing membrane with 500 micron $^{2}$ area and around $100 \mathrm{~nm}$ thick. During the heating cycles to form ncg, this membrane has been strained. The actual part of interest, the freestanding ncg that formed across the hole is much thinner (around $10 \mathrm{~nm}$ ), which necessitates extra care during transfer. A simple crack or deformation of the heating membrane during the transfer would lead to fracture of the ncg film and thus to failure.

After the region of interest (ROI) is selected, Pt/W is electron beam induced deposited (EBID) at one corner (20 um from centre in order to avoid shadowing effect and diffusion-induced artefacts). The size of the lift-out thin film is $50 \mu \mathrm{m}$ x $50 \mu \mathrm{m}$ to avoid any redeposition on the ROI during milling and also acts as safe distance for the ion beam tail. The challenge is not to view the ROI with ion beam as just one snapshot ( 300 ns Dwell time, 1540 x 1200 resolution) would damage the ROI in the ncg.

The shape of the thin film lamella and the length of the FIB cut play an important role in the transfer process. Initially square shaped thin film sections (50 um x $50 \mathrm{um}$ ) where cut by making rectangular cuts of $(50 \mu \mathrm{m} \times 1 \mu \mathrm{m})$. The shape and the cut introduced bending because of strain relaxation in the lamella as shown in figure 1a-c. This bending is a problem for the PTP as the thin film cannot be fixed properly and finally compromises the data of the in situ straining measurements. Therefore, a pentagonal/hexagonal shape (Figure 1d-e) was adapted to successfully reduce the bending. To relieve strain small holes across the corners were drilled before the edge-cut as shown in figure $1 \mathrm{~d}$.

With this approach, bending of the film was minimized and fixing the corners of the film to a PTP was easy with small amounts of EBID-Pt. The transferred thin film was subsequently strained inside the TEM 
using a Bruker in situ TEM holder and the mechanical response was recorded. From the load vs displacement curve figure $2 \mathrm{c}$, we can see that there is no bending in the film and thus confirms that the transfer was successful. It shows that the present method is effective for cutting and transferring highly strained thin films using FIB.
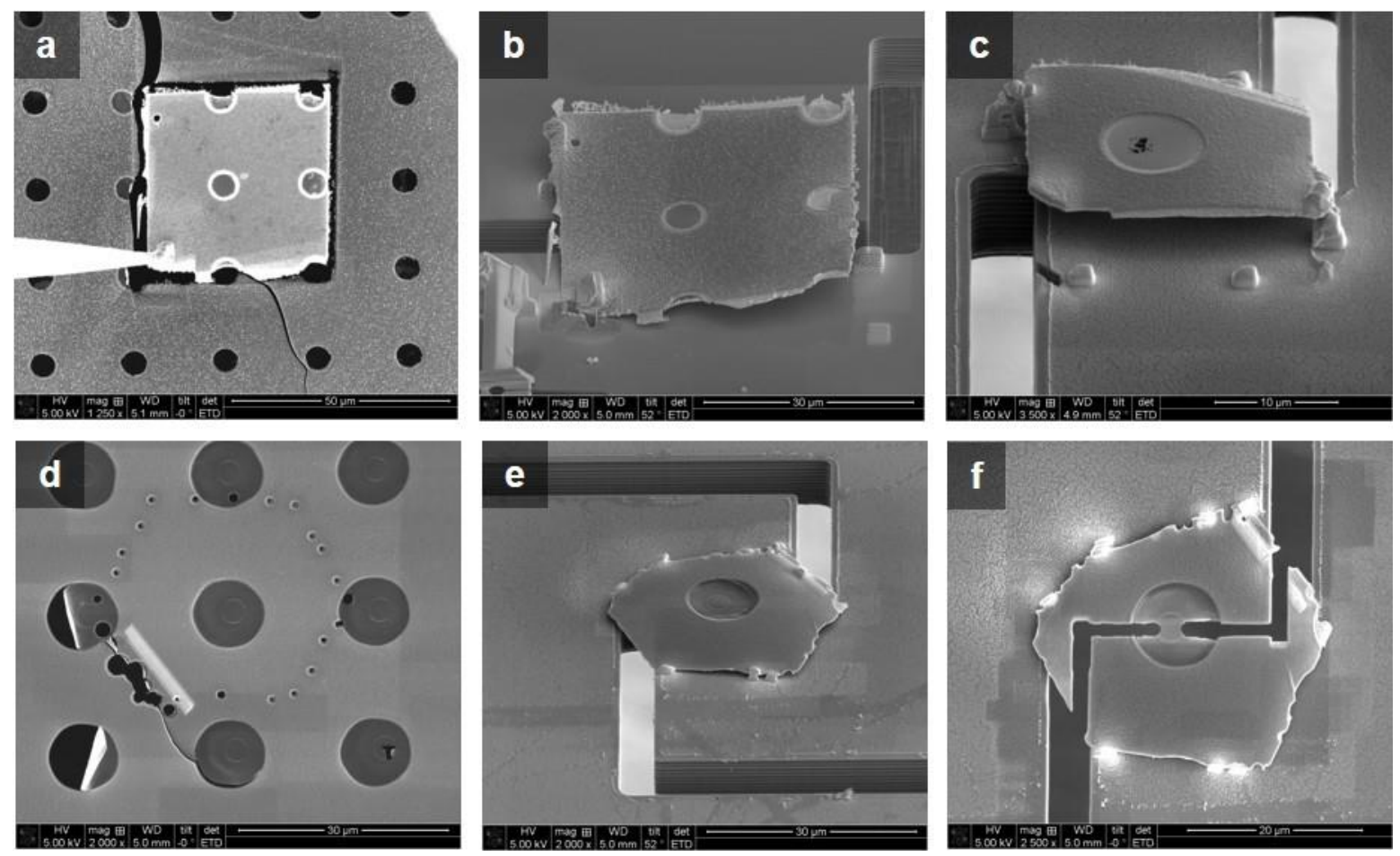

Figure 1. In situ sample preparation of free standing nanocrystalline graphene thin film. (a) square shaped cut; b,c. sample (a) on the PTP device showing bent film; (d) holes drilled in shape of hexagon; e. sample (d) transferred to PTP device; (f) Thin film dog-bone shape for in situ TEM investigation.

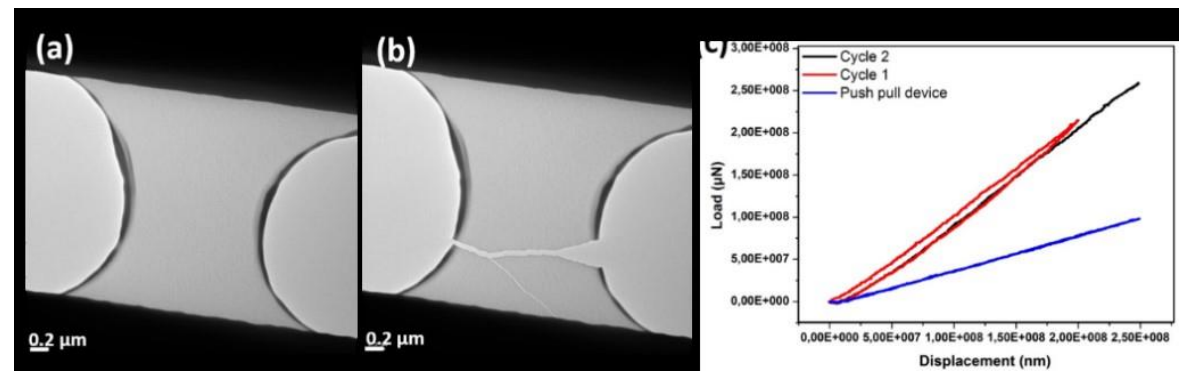

Figure 2. In situ TEM straining of the transferred sample. (a) sample before straining (b) after breakage and (c) load vs displacement graph.

\section{References}

[1] Shyam Kumar, C. N. et al. (2017) 'Understanding the graphitization and growth of free-standing nanocrystalline graphene using in situ transmission electron microscopy’, Nanoscale, 9(35), pp. 12835-12842.

[2] Shyam Kumar, C. N. et al. (2019) 'Nanocrystalline graphene at high temperatures: insight into nanoscale processes', Nanoscale Advances, 1(7), pp. 2485-2494. 